\title{
LIMIT THEOREMS FOR CONTINUOUS-TIME BRANCHING FLOWS
}

\author{
HUI HE, * Beijing Normal University \\ RUGANG MA, ${ }^{* *}$ Central University of Finance and Economics
}

\begin{abstract}
We construct a flow of continuous-time and discrete-state branching processes. Some scaling limit theorems for the flow are proved, which lead to the path-valued branching processes and nonlocal branching superprocesses, over the positive half line, studied in Li (2014).

Keywords: Stochastic flow; branching process; continuous time; superprocess; discrete state; nonlocal branching

2010 Mathematics Subject Classification: Primary 60J68
\end{abstract}

Secondary 60J80; 60G57

\section{Introduction}

A genealogical tree is naturally associated with a Galton-Watson branching process. A continuous-state branching process (CB process) can be obtained as the small particle limit of rescaled Galton-Watson processes; see, e.g. Lamperti (1967). The genealogical structures of binary branching $\mathrm{CB}$ processes were investigated by introducing continuum random trees in the pioneering work of Aldous (1991), (1993). Continuum random trees corresponding to general branching mechanisms were constructed in Le Gall and Le Jan (1998a), (1998b) and were studied further in Duquesne and Le Gall (2002). By pruning a Galton-Watson tree, Aldous and Pitman (1998) and Abraham et al. (2012) constructed a tree-valued Markov process. Treevalued processes associated with general CB processes were studied in Abraham and Delmas (2012) by using pruning arguments.

Motivated by the study of genealogy trees for critical branching processes conditioned on nonextinction, Bakhtin (2011) studied a flow of binary branching continuous-state branching processes with immigration driven by a time-space Gaussian white noise. He also pointed out the connection of the model with a superprocess conditioned on nonextinction. In $\mathrm{Li}$ (2014), a class of path-valued branching processes were constructed and studied using the techniques of stochastic equations and superprocesses. The work is closely related to those of Bertoin and Le Gall (2006) and Dawson and $\mathrm{Li}$ (2014). In a special case, the path-valued branching processes in $\mathrm{Li}$ (2014) can be coded by the tree-valued processes of Abraham and Delmas (2012). In $\mathrm{He}$ and $\mathrm{Ma}$ (2014), two flows of discrete time and state Galton-Watson branching processes were introduced. There it was showed that suitable rescaled sequences of those flows converge to special forms of the flows of Dawson and Li (2012) and Li (2014). The limit theorems in $\mathrm{He}$ and $\mathrm{Ma}$ (2014) were given in the setting of the corresponding superprocesses. From those

Received 17 April 2012; revision received 27 September 2012.

* Postal address: Laboratory of Mathematics and Complex Systems, School of Mathematical Sciences, Beijing Normal University, Beijing 100875, People's Republic of China. Email address: hehui@bnu.edu.cn

** Postal address: School of Applied Mathematics, Central University of Finance and Economics, Beijing 100081, People's Republic of China. Email address: marugang@mail.bnu.edu.cn 
limit theorems, the convergence of the finite-dimensional distributions of the corresponding path-valued processes was derived. The results give a better understanding of the connection between discrete and continuum tree-valued branching processes.

In this paper we introduce flows of continuous-time and discrete-state branching processes. We shall prove the scaling limit theorems, of the type given in He and Ma (2014), for those flows. In Section 2, a short review is given of the path-valued branching processes and nonlocal branching superprocesses studied in Li (2014). In Section 3 we construct a continuous-time and discrete-state branching process as the strong solution of a stochastic integral equation. In Section 4, the construction is extended to branching flows by considering systems of stochastic equations. In Section 5 we prove that suitable rescaled sequences of those flows converge to the nonlocal branching superprocess. From the limit theorem we also derive the convergence of the finite-dimensional distributions of the corresponding path-valued processes.

Let $\mathbb{N}=\{0,1,2, \ldots\}$ and $\mathbb{N}_{+}=\{1,2, \ldots\}$. Let $M[0,1]$ be the set of finite Borel measures on $[0,1]$ endowed with the topology of weak convergence. We identify $M[0,1]$ with the set $F[0,1]$ of positive right continuous increasing functions on $[0,1]$. Let $B[0,1]$ be the Banach space of bounded Borel functions on $[0,1]$ endowed with the supremum norm $\|\cdot\|$. Let $C[0,1]$ denote its subspace of continuous functions. We use $B[0,1]^{+}$and $C[0,1]^{+}$to denote the subclasses of nonnegative elements and $C[0,1]^{++}$to denote the subset of $C[0,1]^{+}$of functions bounded away from 0 . For $\mu \in M[0,1]$ and $f \in B[0,1]$, we write $\langle\mu, f\rangle=\int f \mathrm{~d} \mu$ if the integral exists. Let $D([0, \infty), M[0,1])$ denote the space of càdlàg paths from $[0, \infty)$ to $M[0,1]$ endowed with the Skorokhod topology. Throughout this paper we only consider continuous-time processes, so we shall often omit this phrase in the sequel.

\section{Preliminaries}

In this section we recall some results established in $\mathrm{Li}$ (2014) on flows of CB processes and nonlocal branching superprocesses over the positive half line. By a branching mechanism $\phi$ we mean a function $\phi$ on $[0, \infty)$ with the representation

$$
\phi(z)=b z+\frac{1}{2} \sigma^{2} z^{2}+\int_{0}^{\infty}\left(\mathrm{e}^{-z u}-1+z u\right) m(\mathrm{~d} u),
$$

where $\sigma \geq 0$ and $b$ are constants and $\left(u \wedge u^{2}\right) m(\mathrm{~d} u)$ is a finite measure on $(0, \infty)$. Consider a family of branching mechanisms $\left\{\phi_{q}: q \in[0,1]\right\}$ that is admissible in the sense that each $\phi_{q}$ is given by (2.1) with parameters $(b, m)=\left(b_{q}, m_{q}\right)$ depending on $q \in[0,1]$ and, for each $z \geq 0$, the function $q \mapsto \phi_{q}(z)$ is decreasing and continuously differentiable with the derivative $\psi_{\theta}(z)=-(\partial / \partial \theta) \phi_{\theta}(z)$ of the form

$$
\psi_{\theta}(z)=h_{\theta} z+\int_{0}^{\infty}\left(1-\mathrm{e}^{-z u}\right) n_{\theta}(\mathrm{d} u)
$$

where $h_{\theta} \geq 0$ and $n_{\theta}(\mathrm{d} u)$ is a $\sigma$-finite kernel from $[0,1]$ to $(0, \infty)$ satisfying

$$
\sup _{0 \leq \theta \leq 1}\left[h_{\theta}+\int_{0}^{\infty} u n_{\theta}(\mathrm{d} u)\right]<\infty .
$$

Let $m(\mathrm{~d} z, \mathrm{~d} \theta)$ be the measure on $(0, \infty) \times[0,1]$ defined by

$$
m([c, d] \times[0, q])=m_{q}[c, d], \quad q \in[0,1], d>c>0 .
$$


Let $W(\mathrm{~d} s, \mathrm{~d} u)$ be a white noise on $(0, \infty)^{2}$ based on the Lebesgue measure, and let

$$
\tilde{N}(\mathrm{~d} s, \mathrm{~d} z, \mathrm{~d} \theta, \mathrm{d} u)
$$

be a compensated Poisson random measure on $(0, \infty)^{2} \times[0,1] \times(0, \infty)$ with intensity $\mathrm{d} s m(\mathrm{~d} z, \mathrm{~d} \theta) \mathrm{d} u$. By the results in Li (2014), the stochastic equation

$$
\begin{aligned}
Y_{t}(q)= & Y_{0}(q)-b_{q} \int_{0}^{t} Y_{s-}(q) \mathrm{d} s+\sigma \int_{0}^{t} \int_{0}^{Y_{s-}(q)} W(\mathrm{~d} s, \mathrm{~d} u) \\
& +\int_{0}^{t} \int_{0}^{\infty} \int_{[0, q]} \int_{0}^{Y_{s-}(q)} z \tilde{N}(\mathrm{~d} s, \mathrm{~d} z, \mathrm{~d} \theta, \mathrm{d} u)
\end{aligned}
$$

has a unique solution flow $\left\{Y_{t}(q): t \geq 0, q \in[0,1]\right\}$. For each $q \in[0,1]$, the one-dimensional process $\left\{Y_{t}(q): t \geq 0\right\}$ is a CB process with branching mechanism $\phi_{q}$. The flow is increasing in $q \in[0,1]$. It was verified in $\mathrm{Li}(2014)$ that $\left\{\left(Y_{t}(q)\right)_{t \geq 0}: q \in[0,1]\right\}$ can be identified as a pathvalued branching process. Moreover, the flow induces a càdlàg $M[0,1]$-valued superprocess $\left\{Y_{t}: t \geq 0\right\}$ which is the unique solution of the following martingale problem: for every $G \in C^{2}(\mathbb{R})$ and $f \in C[0,1]$,

$$
\begin{aligned}
G\left(\left\langle Y_{t}, f\right\rangle\right)= & G\left(\left\langle Y_{0}, f\right\rangle\right)+\int_{0}^{t} G^{\prime}\left(\left\langle Y_{s}, f\right\rangle\right) \mathrm{d} s \int_{[0,1]} Y_{s}(\mathrm{~d} x) \int_{[0,1]} f(x \vee \theta) h_{\theta} \mathrm{d} \theta \\
& -b_{0} \int_{0}^{t} G^{\prime}\left(\left\langle Y_{s}, f\right\rangle\right)\left\langle Y_{s}, f\right\rangle \mathrm{d} s+\frac{1}{2} \sigma^{2} \int_{0}^{t} G^{\prime \prime}\left(\left\langle Y_{s}, f\right\rangle\right)\left\langle Y_{s}, f^{2}\right\rangle \mathrm{d} s \\
& +\int_{0}^{t} \mathrm{~d} s \int_{[0,1]} Y_{s}(\mathrm{~d} x) \int_{0}^{\infty}\left[G\left(\left\langle Y_{s}, f\right\rangle+z f(x)\right)-G\left(\left\langle Y_{s}, f\right\rangle\right)\right. \\
& +\int_{0}^{t} \mathrm{~d} s \int_{[0,1]} Y_{s}(\mathrm{~d} x) \int_{[0,1]} \mathrm{d} \theta \int_{0}^{\infty}\left[G\left(\left\langle Y_{s}, f\right\rangle+z f(x \vee \theta)\right)-G\left(\left\langle Y_{s}, f\right\rangle\right)\right] \\
& \times n_{\theta}(\mathrm{d} z)+\text { local martingale. }
\end{aligned}
$$

Let $f \mapsto \Psi(\cdot, f)$ be the operator on $C^{+}[0,1]$ defined by

$$
\Psi(x, f)=\int_{[0,1]} f(x \vee \theta) h_{\theta} \mathrm{d} \theta+\int_{[0,1]} \mathrm{d} \theta \int_{0}^{\infty}\left(1-\mathrm{e}^{-z f(x \vee \theta)}\right) n_{\theta}(\mathrm{d} z) .
$$

Then the superprocess $\left\{Y_{t}: t \geq 0\right\}$ has local branching mechanism $\phi_{0}$ and nonlocal branching mechanism $\Psi$. The transition semigroup $\left(Q_{t}\right)_{t \geq 0}$ of $\left\{Y_{t}: t \geq 0\right\}$ is given by

$$
\int_{M[0,1]} \mathrm{e}^{-\langle\nu, f\rangle} Q_{t}(\mu, \mathrm{d} \nu)=\exp \left\{-\left\langle\mu, V_{t} f\right\rangle\right\}, \quad f \in C^{+}[0,1],
$$

where $t \mapsto V_{t} f$ is the unique locally bounded positive solution of

$$
V_{t} f(x)=f(x)-\int_{0}^{t}\left[\phi_{0}\left(V_{s} f(x)\right)-\Psi\left(x, V_{s} f\right)\right] \mathrm{d} s, \quad t \geq 0, x \in[0,1] .
$$

We refer the reader to $\mathrm{Li}$ (2014) for the derivations of the superprocess $\left\{Y_{t}: t \geq 0\right\}$.

Remark 2.1. Usually, we may only use the Lévy measure $m$ in $\phi$ in (2.1) to integrate $1 \wedge u^{2}$, and an indicator function is added in the integral. In fact, the assumption that $\left(u \wedge u^{2}\right) m(d u)$ is a finite measure on $(0, \infty)$ is equivalent to $\phi$ being locally Lipschitz; see Proposition 1.45 
of Li (2011). This assumption is technically required to construct the scaling limits of GaltonWatson processes; see Proposition 3.40 of Li (2011) and Assumption 5.1 below.

\section{Stochastic equations for discrete-state branching processes}

In this section we give a construction of the continuous-time and discrete-state branching process as the solution of a stochastic integral equation driven by a Poisson random measure. Stochastic integral equations of this type were used in $\mathrm{Li}$ and $\mathrm{Ma}$ (2008) to construct catalytic branching processes. We here give all the details for completeness.

Let $g=g(z)=\sum_{i=0}^{\infty} p_{i} z^{i}$ be a probability generating function with $g^{\prime}(1)<\infty$. Let $N(\mathrm{~d} s, \mathrm{~d} z, \mathrm{~d} u)$ be a Poisson random measure on $(0, \infty) \times \mathbb{N} \times(0, \infty)$ with intensity $\sigma \mathrm{d} s \pi(\mathrm{d} z) \mathrm{d} u$, where $\sigma>0$ is a constant and $\pi(\mathrm{d} z):=\sum_{i=0}^{\infty} p_{i} \delta_{i}(\mathrm{~d} z)$. Suppose that $X_{0}$ is a nonnegative integer-valued random variable satisfying $\mathbb{E}\left[X_{0}\right]<\infty$. We assume that $X_{0}$ is independent of $N(\mathrm{~d} s, \mathrm{~d} z, \mathrm{~d} u)$ and consider the stochastic integral equation

$$
X_{t}=X_{0}+\int_{0}^{t} \int_{\mathbb{N}} \int_{0}^{X_{s-}}(z-1) N(\mathrm{~d} s, \mathrm{~d} z, \mathrm{~d} u) .
$$

By a solution of (3.1) we mean a nonnegative càdlàg progressive process $\left\{X_{t}: t \geq 0\right\}$ satisfying the equation almost surely (a.s.) for each $t \geq 0$. We say pathwise uniqueness of solution holds for (3.1) if any two solutions of the equation, with the same initial state, are indistinguishable.

Theorem 3.1. Suppose that $\left\{X_{t}^{1}\right\}$ and $\left\{X_{t}^{2}\right\}$ are two solutions of (3.1) satisfying the inequality $\mathbb{E}\left[\left|X_{0}^{1}+X_{0}^{2}\right|\right]<\infty$. Then we have

$$
\mathbb{E}\left[\left|X_{t}^{2}-X_{t}^{1}\right|\right] \leq \mathbb{E}\left[\left|X_{0}^{2}-X_{0}^{1}\right|\right] \exp \left\{\sigma t\left(g^{\prime}(1)+1\right)\right\} .
$$

Consequently, the pathwise uniqueness of solution holds for (3.1).

Proof. The pathwise uniqueness for (3.1) follows from Theorem 2.1 of Dawson and Li (2012). We present a proof of the result here for completeness. Let $\xi_{t}=X_{t}^{2}-X_{t}^{1}$ for $t \geq 0$. From (3.1) we have

$$
\begin{aligned}
\xi_{t}= & X_{0}^{2}-X_{0}^{1}+\int_{0}^{t} \int_{\mathbb{N}} \int_{X_{s-}^{1}}^{X_{s-}^{2}}(z-1) \mathbf{1}_{\left\{X_{s-}^{1} \leq X_{s-}^{2}\right\}} N(\mathrm{~d} s, \mathrm{~d} z, \mathrm{~d} u) \\
& -\int_{0}^{t} \int_{\mathbb{N}} \int_{X_{s-}^{2}}^{X_{s-}^{1}}(z-1) \mathbf{1}_{\left\{X_{s-}^{1}>X_{s-}^{2}\right\}} N(\mathrm{~d} s, \mathrm{~d} z, \mathrm{~d} u) .
\end{aligned}
$$

Let $\tau_{m}=\inf \left\{t \geq 0: X_{t}^{1} \geq m\right.$ or $\left.X_{t}^{2} \geq m\right\}$. Then we have

$$
\begin{aligned}
\mathbb{E}\left[\left|\xi_{t \wedge \tau_{m}}\right|\right] \leq & \mathbb{E}\left[\left|\xi_{0}\right|\right]+\mathbb{E} \int_{0}^{t \wedge \tau_{m}} \int_{\mathbb{N}} \int_{X_{s-}^{1}}^{X_{s-}^{2}}(z+1) \mathbf{1}_{\left\{X_{s-}^{1} \leq X_{s-}^{2}\right\}} N(\mathrm{~d} s, \mathrm{~d} z, \mathrm{~d} u) \\
& +\mathbb{E} \int_{0}^{t \wedge \tau_{m}} \int_{\mathbb{N}} \int_{X_{s-}^{2}}^{X_{s-}^{1}}(z+1) \mathbf{1}_{\left\{X_{s-}^{1}>X_{s-}^{2}\right\}} N(\mathrm{~d} s, \mathrm{~d} z, \mathrm{~d} u) \\
= & \mathbb{E}\left[\left|\xi_{0}\right|\right]+\mathbb{E} \int_{0}^{t \wedge \tau_{m}} \mathrm{~d} s \int_{\mathbb{N}} \xi_{s-} \mathbf{1}_{\left\{\xi_{s-} \geq 0\right\}}(z+1) \sigma \pi(\mathrm{d} z) \\
& +\mathbb{E} \int_{0}^{t \wedge \tau_{m}} \mathrm{~d} s \int_{\mathbb{N}}\left(-\xi_{s-}\right) \mathbf{1}_{\left\{\xi_{s-}<0\right\}}(z+1) \sigma \pi(\mathrm{d} z) \\
\leq & \mathbb{E}\left[\left|\xi_{0}\right|\right]+\int_{0}^{t} \mathbb{E}\left[\left|\xi_{s \wedge \tau_{m}}\right|\right] \sigma\left(g^{\prime}(1)+1\right) \mathrm{d} s
\end{aligned}
$$


By Gronwall's inequality we obtain

$$
\mathbb{E}\left[\left|\xi_{t \wedge \tau_{m}}\right|\right] \leq \mathbb{E}\left[\left|\xi_{0}\right|\right] \exp \left\{\sigma t\left(g^{\prime}(1)+1\right)\right\} .
$$

Then (3.2) follows by Fatou's lemma, completing the proof.

By Theorem 2.5 of Dawson and Li (2012), there is a unique strong solution to (3.1). Here we give a simple direct proof of the existence of the solution. We first take an $n \in \mathbb{N}_{+}$and consider the following stochastic equation

$$
X_{t}=X_{0}+\int_{0}^{t} \int_{\mathbb{N}} \int_{0}^{X_{s-\wedge n}}(z-1) N(\mathrm{~d} s, \mathrm{~d} z, \mathrm{~d} u)
$$

Proposition 3.1. For each $n \geq 1$, there is a solution $\left\{X_{t}^{n}: t \geq 0\right\}$ of (3.3).

Proof. Let $\left\{S_{k}: k=1,2, \ldots\right\}$ be the set of jump times of the Poisson process

$$
t \mapsto \int_{0}^{t} \int_{\mathbb{N}} \int_{0}^{n} N(\mathrm{~d} s, \mathrm{~d} z, \mathrm{~d} u)
$$

We clearly have $S_{k} \rightarrow \infty$ as $k \rightarrow \infty$. For $0 \leq t<S_{1}$, set $X_{t}^{n}=X_{0}$. Suppose that $X_{t}^{n}$ has been defined for $0 \leq t<S_{k}$ and let

$$
X_{t}^{n}=X_{S_{k}-}^{n}+\int_{\left\{S_{k}\right\}} \int_{\mathbb{N}} \int_{0}^{X_{S_{k}-}^{n} \wedge n}(z-1) N(\mathrm{~d} s, \mathrm{~d} z, \mathrm{~d} u), \quad S_{k} \leq t<S_{k+1} .
$$

From the construction of $X_{S_{k}}^{n}$ we see that $X_{S_{k}}^{n}-X_{S_{k-1}}^{n} \geq-1$. Furthermore, since $X_{S_{k-1}}^{n}=0$ implies that $X_{S_{k}}^{n}=0$, we have $X_{S_{k}}^{n} \in \mathbb{N}$. By induction we have defined a nonnegative process $\left\{X_{t}^{n}: t \geq 0\right\}$ which is clearly a solution to (3.3).

Proposition 3.2. Let $\left\{X_{t}^{n}\right\}$ be a solution of (3.3). Then we have

$$
\mathbb{E}\left[\sup _{0 \leq s \leq t} X_{s}^{n}\right] \leq \mathbb{E}\left[X_{0}\right] \exp \left\{\sigma g^{\prime}(1) t\right\}, \quad t \geq 0 .
$$

Proof. From (3.3) we have

$$
\begin{aligned}
\mathbb{E}\left[\sup _{0 \leq s \leq t} X_{s}^{n}\right] & \leq \mathbb{E}\left[X_{0}\right]+\mathbb{E}\left[\int_{0}^{t} \int_{\mathbb{N}} \int_{0}^{X_{s-\wedge}^{n}} z N(\mathrm{~d} s, \mathrm{~d} z, \mathrm{~d} u)\right] \\
& =\mathbb{E}\left[X_{0}\right]+\mathbb{E}\left[\int_{0}^{t} \mathrm{~d} s \int_{\mathbb{N}}\left(X_{s-\wedge}^{n} \wedge n\right) z \sigma \pi(\mathrm{d} z)\right]
\end{aligned}
$$

Thus $t \mapsto \mathbb{E}\left[\sup _{0 \leq s \leq t} X_{s}^{n}\right]$ is a locally bounded function. Moreover,

$$
\begin{aligned}
\mathbb{E}\left[\sup _{0 \leq s \leq t} X_{s}^{n}\right] & \leq \mathbb{E}\left[X_{0}\right]+\int_{0}^{t} \mathrm{~d} s \int_{\mathbb{N}} \mathbb{E}\left[\sup _{0 \leq r \leq s} X_{r}^{n}\right] z \sigma \pi(\mathrm{d} z) \\
& =\mathbb{E}\left[X_{0}\right]+\sigma g^{\prime}(1) \int_{0}^{t} \mathbb{E}\left[\sup _{0 \leq r \leq s} X_{r}^{n}\right] \mathrm{d} s .
\end{aligned}
$$

By Gronwall's lemma we obtain the result, completing the proof. 
By a modification of the proof of Theorem 3.1 we obtain the following Proposition.

Proposition 3.3. Suppose that $\left\{X_{t}^{n, 1}\right\}$ and $\left\{X_{t}^{n, 2}\right\}$ are two solutions of (3.3). Then we have

$$
\mathbb{E}\left[\left|X_{t}^{n, 2}-X_{t}^{n, 1}\right|\right] \leq \mathbb{E}\left[\left|X_{0}^{n, 2}-X_{0}^{n, 1}\right|\right] \exp \left\{\sigma t\left(g^{\prime}(1)+1\right)\right\}
$$

Consequently, the pathwise uniqueness of solution holds for (3.3).

Proposition 3.4. Let $\left\{X_{t}^{n}: t \geq 0\right\}$ be the solution of (3.3) with $n=1,2, \ldots$. Then the sequence $\left\{X_{t}^{n}: t \geq 0\right\}$ is tight in $D([0, \infty), \mathbb{N})$.

Proof. By Proposition 3.2, it is easy to see that

$$
t \mapsto C_{t}:=\sup _{n \geq 1} \mathbb{E}\left[\sup _{0 \leq s \leq t} X_{s}^{n}\right]
$$

is locally bounded. Then, for every fixed $t \geq 0$, the sequence of random variables $X_{t}^{n}$ is tight. Moreover, in view of (3.3), if $\left\{\tau_{n}\right\}$ is a sequence of stopping times bounded above by $T \geq 0$, we have

$$
\begin{aligned}
\mathbb{E}\left[\left|X_{t+\tau_{n}}^{n}-X_{\tau_{n}}^{n}\right|\right] & =\mathbb{E}\left[\left|\int_{\tau_{n}}^{t+\tau_{n}} \int_{\mathbb{N}} \int_{0}^{X_{s-}^{n} \wedge n}(z-1) N(\mathrm{~d} s, \mathrm{~d} z, \mathrm{~d} u)\right|\right] \\
& \leq \mathbb{E}\left[\int_{0}^{t} \mathrm{~d} s \int_{\mathbb{N}}\left(X_{\left(s+\tau_{n}\right)-}^{n} \wedge n\right)(z+1) \sigma \pi(\mathrm{d} z)\right] \\
& \leq \sigma\left(g^{\prime}(1)+1\right) \int_{0}^{t} \mathbb{E}\left[X_{\left(s+\tau_{n}\right)-}^{n}\right] \mathrm{d} s \\
& \leq \mathbb{E}\left[X_{0}\right] \exp \left\{\sigma g^{\prime}(1)(t+T)\right\} \sigma\left(g^{\prime}(1)+1\right) t
\end{aligned}
$$

where the last inequality follows by Proposition 3.2. Consequently, as $t \rightarrow 0$,

$$
\sup _{n \geq 1} \mathbb{E}\left[\left|X_{t+\tau_{n}}^{n}-X_{\tau_{n}}^{n}\right|\right] \rightarrow 0 \text {. }
$$

Then $\left\{X_{t}^{n}: t \geq 0\right\}$ is tight in $D([0, \infty), \mathbb{N})$ by the criterion of Aldous (1978); see also Ethier and Kurtz (1986, pp. 137-138).

Theorem 3.2. There is a solution $\left\{X_{t}: t \geq 0\right\}$ of (3.1).

Proof. For each $n \geq 1$, let $\left\{X_{t}^{n}: t \geq 0\right\}$ be the solution of (3.3). Furthermore, define $\tau_{n}=\inf \left\{t \geq 0: X_{t}^{n} \geq n\right\}$. From Proposition 3.2, it follows that

$$
\mathbb{E}\left[X_{t \wedge \tau_{n}}^{n}\right] \leq \mathbb{E}\left[\sup _{0 \leq s \leq t} X_{s}^{n}\right] \leq \mathbb{E}\left[X_{0}\right] \exp \left\{\sigma g^{\prime}(1) t\right\}, \quad t \geq 0 .
$$

Then we have

$$
\mathbb{E}\left[X_{t \wedge \tau_{n}}^{n} \mathbf{1}_{\left\{\tau_{n} \leq t\right\}}\right] \leq \mathbb{E}\left[X_{0}\right] \exp \left\{\sigma g^{\prime}(1) t\right\} .
$$

By the right continuity of $\left\{X_{t}^{n}\right\}$ we have $X_{\tau_{n}}^{n} \geq n$, so

$$
n \mathbb{P}\left[\left\{\tau_{n} \leq t\right\}\right] \leq \mathbb{E}\left[X_{0}\right] \exp \left\{\sigma g^{\prime}(1) t\right\}, \quad t \geq 0 .
$$

This implies that $\tau_{n} \rightarrow \infty$ almost surely as $n \rightarrow \infty$. On the other hand, $\left\{X_{t}^{n}\right\}$ satisfies (3.1) for $0 \leq t<\tau_{n}$. By the pathwise uniqueness of the solution of (3.1) we obtain, for any $i, j \in \mathbb{N}$,

$$
X_{t}^{i}=X_{t}^{j}, \quad t<\tau_{i} \wedge \tau_{j}
$$

Let $\left\{X_{t}\right\}$ be the process such that $X_{t}=X_{t}^{n}$ for all $0 \leq t<\tau_{n}$ and $n \geq 1$. It is easily seen that $\left\{X_{t}\right\}$ is a solution of (3.1). This completes the proof. 
Theorem 3.1 and Theorem 3.2 imply that (3.1) has a unique strong solution and the solution $\left\{X_{t}: t \geq 0\right\}$ is a strong Markov process; see, e.g. Ikeda and Watanabe (1989, pp. 163-166, 215). Let $B(\mathbb{N})$ denote the set of bounded functions on $\mathbb{N}$. By Itô's formula, it is easy to see that $\left\{X_{t}: t \geq 0\right\}$ has a generator $A$ defined by

$$
A f(x)=\sigma x \sum_{i=0}^{\infty}[f(x+i-1)-f(x)] p_{i}, \quad x \in \mathbb{N}, f \in B(\mathbb{N}) .
$$

Hence, $\left\{X_{t}: t \geq 0\right\}$ is a Galton-Watson branching process with $\sigma$-exponentially distributed life time and offspring distribution $\left\{p_{i}: i \geq 0\right\}$.

In fact, let $N^{(1)}(\mathrm{d} s, \mathrm{~d} z, \mathrm{~d} u)$ and $N^{(2)}(\mathrm{d} s, \mathrm{~d} z, \mathrm{~d} u)$ be two mutually independent Poisson random measures on $(0, \infty) \times \mathbb{N} \times(0, \infty)$ with the same intensity $\sigma \mathrm{d} s \pi(\mathrm{d} z) \mathrm{d} u$. Consider the two stochastic equations

$$
X_{t}^{(1)}=X_{0}^{(1)}+\int_{0}^{t} \int_{\mathbb{N}} \int_{0}^{X_{s-}^{(1)}}(z-1) N^{(1)}(\mathrm{d} s, \mathrm{~d} z, \mathrm{~d} u)
$$

and

$$
X_{t}^{(2)}=X_{0}^{(2)}+\int_{0}^{t} \int_{\mathbb{N}} \int_{0}^{X_{s-}^{(2)}}(z-1) N^{(2)}(\mathrm{d} s, \mathrm{~d} z, \mathrm{~d} u) .
$$

Clearly, $X_{t}^{(1)}$ and $X_{t}^{(2)}$ are mutually independent. Set $X_{t}=X_{t}^{(1)}+X_{t}^{(2)}$. Since the random measure

$$
N^{\prime}(\mathrm{d} s, \mathrm{~d} z):=\int_{\left\{0<u \leq X_{s-}^{(1)}\right\}} N^{(1)}(\mathrm{d} s, \mathrm{~d} z, \mathrm{~d} u)+\int_{\left\{0<u \leq X_{s-}^{(2)}\right\}} N^{(2)}(\mathrm{d} s, \mathrm{~d} z, \mathrm{~d} u)
$$

has the predictable compensator $\sigma X_{s-} \mathrm{d} s \pi(\mathrm{d} z)$, by representation theorems for semimartingales, on an extension of the original probability space, there is a Poisson random measure on $(0, \infty) \times \mathbb{N} \times(0, \infty)$ with intensity $\sigma \mathrm{d} s \pi(\mathrm{d} z) \mathrm{d} u$ such that

$$
X_{t}=X_{0}+\int_{0}^{t} \int_{\mathbb{N}} \int_{0}^{X_{s-}}(z-1) N(\mathrm{~d} s, \mathrm{~d} z, \mathrm{~d} u)
$$

see, e.g. Ikeda and Watanabe (1989, p. 93). Hence, the solution of (3.1) is a branching process (continuous time and discrete state). This gives another derivation of the branching property of $\left\{X_{t}: t \geq 0\right\}$.

\section{The flow of discrete-state branching processes}

In this section we give a formulation of the discrete-state branching flow as the solution flow of a set of stochastic integral equations. Let $\left\{g_{\theta}: \theta \geq 0\right\}$ be a family of probability generating functions, that is, for each $\theta \geq 0$,

$$
g_{\theta}(z)=\sum_{i=0}^{\infty} p_{i}(\theta) z^{i}, \quad|z| \leq 1
$$

where $p_{i}(\theta) \geq 0$ and $\sum_{k=0}^{\infty} p_{i}(\theta)=1$. Moreover, we assume that $\theta \mapsto g_{\theta}^{\prime}(1)$ is continuous and $p_{i}\left(\theta_{2}\right) \geq p_{i}\left(\theta_{1}\right)$ holds for all $\theta_{2} \geq \theta_{1} \geq 0$ and $i \in \mathbb{N}_{+}$. Define a family of probability measures $\left\{\pi_{\theta}: \theta \geq 0\right\}$ on $\mathbb{N}$ by

$$
\pi_{\theta}(\mathrm{d} z)=\sum_{i=0}^{\infty} p_{i}(\theta) \delta_{i}(\mathrm{~d} z) .
$$


Then we have $\pi_{\theta_{2}}{\mid \mathbb{N}_{+}} \geq\left.\pi_{\theta_{1}}\right|_{\mathbb{N}_{+}}$for all $\theta_{2} \geq \theta_{1} \geq 0$. Let $\bar{\pi}(\mathrm{d} z, \mathrm{~d} \theta)$ be the measure on $\mathbb{N}_{+} \times$ $[0, \infty)$ defined by

$$
\bar{\pi}(A \times[0, \theta])=\pi_{\theta}(A), \quad A \subset \mathbb{N}_{+}, \theta \geq 0 .
$$

Note that the positive function $\theta \mapsto b(\theta):=\pi_{\theta}(\{0\})$ is decreasing.

Let $q \mapsto X_{0}(q)$ be a deterministic, nonnegative, right-continuous, nondecreasing function on $[0, \infty)$ that takes values in $\mathbb{N}$. Let $N(\mathrm{~d} s, \mathrm{~d} z, \mathrm{~d} \theta, \mathrm{d} u)$ be a Poisson random measure on $(0, \infty) \times \mathbb{N} \times[0, \infty) \times(0, \infty)$ with intensity $\sigma \mathrm{d} s \bar{\pi}(\mathrm{d} z, \mathrm{~d} \theta) \mathrm{d} u$ and $N_{0}(\mathrm{~d} s, \mathrm{~d} \theta, \mathrm{d} u)$ a Poisson random measure on $(0, \infty)^{3}$ with intensity $\sigma \mathrm{d} s \mathrm{~d} \theta \mathrm{d} u$. Suppose that $N(\mathrm{~d} s, \mathrm{~d} z, \mathrm{~d} \theta, \mathrm{d} u)$ and $N_{0}(\mathrm{~d} s, \mathrm{~d} \theta, \mathrm{d} u)$ are independent of each other. Consider the stochastic integral equation

$$
\begin{aligned}
X_{t}(q)= & X_{0}(q)+\int_{0}^{t} \int_{\mathbb{N}_{+}} \int_{[0, q]} \int_{0}^{X_{s-}(q)}(z-1) N(\mathrm{~d} s, \mathrm{~d} z, \mathrm{~d} \theta, \mathrm{d} u) \\
& -\int_{0}^{t} \int_{0}^{b(q)} \int_{0}^{X_{s-}(q)} N_{0}(\mathrm{~d} s, \mathrm{~d} \theta, \mathrm{d} u)
\end{aligned}
$$

Note that, for each $q \geq 0$,

$$
\int_{\{0<\theta \leq b(q)\}} N_{0}(\mathrm{~d} s, \mathrm{~d} \theta, \mathrm{d} u)
$$

is a Poisson random measure with intensity $\sigma b(q) \mathrm{d} s \mathrm{~d} u=\sigma \bar{\pi}_{0}(\mathbb{N} \times[0, q]) \mathrm{d} s \mathrm{~d} u$, where $\bar{\pi}_{0}(\mathrm{~d} z, \mathrm{~d} \theta)$ is a measure on $\mathbb{N} \times[0, \infty)$ defined by

$$
\bar{\pi}_{0}(A \times[0, q])=\pi_{q}(\{0\}) \delta_{0}(A), \quad A \subset \mathbb{N}, \theta \geq 0 .
$$

By representation theorems for semimartingales, there exists a Poisson random measure $N_{1}(\mathrm{~d} s, \mathrm{~d} z, \mathrm{~d} \theta, \mathrm{d} u)$ on $(0, \infty) \times \mathbb{N} \times[0, \infty) \times(0, \infty)$ with intensity $\sigma \mathrm{d} s \bar{\pi}_{0}(\mathrm{~d} z, \mathrm{~d} \theta) \mathrm{d} u$ such that, for every $E \in \mathcal{B}(0, \infty)$,

$$
\int_{0}^{t} \int_{0}^{b(q)} \int_{E} N_{0}(\mathrm{~d} s, \mathrm{~d} \theta, \mathrm{d} u)=\int_{0}^{t} \int_{\mathbb{N}} \int_{[0, q]} \int_{E} N_{1}(\mathrm{~d} s, \mathrm{~d} z, \mathrm{~d} \theta, \mathrm{d} u) ;
$$

see, e.g. Ikeda and Watanabe (1989, p. 93). Define $N_{2}(\mathrm{~d} s, \mathrm{~d} z, \mathrm{~d} u)$ by

$$
N_{2}(\mathrm{~d} s, \mathrm{~d} z, \mathrm{~d} u)=\int_{\{0 \leq \theta \leq q\}} N(\mathrm{~d} s, \mathrm{~d} z, \mathrm{~d} \theta, \mathrm{d} u)+\int_{\{0 \leq \theta \leq q\}} N_{1}(\mathrm{~d} s, \mathrm{~d} z, \mathrm{~d} \theta, \mathrm{d} u) .
$$

Then $N_{2}$ is a Poisson random measure on $(0, \infty) \times \mathbb{N} \times(0, \infty)$ with intensity $\sigma \mathrm{d} s \pi_{q}(\mathrm{~d} z) \mathrm{d} u$, and (4.1) can be rewritten as

$$
X_{t}(q)=X_{0}(q)+\int_{0}^{t} \int_{\mathbb{N}} \int_{0}^{X_{s-}(q)}(z-1) N_{2}(\mathrm{~d} s, \mathrm{~d} z, \mathrm{~d} u) .
$$

By Theorem 3.2 we see that, for each $q \geq 0,(4.1)$ has a unique strong solution $\left\{X_{t}(q): t \geq 0\right\}$.

Theorem 4.1. Suppose that $q \geq p \geq 0$. Let $\left\{X_{t}(q)\right\}$ be the solution of (4.1) and $\left\{X_{t}(p)\right\}$ be the solution of the equation with $q$ replaced by $p$. Then we have

$$
\mathbb{P}\left\{X_{t}(q) \geq X_{t}(p) \text { for all } t \geq 0\right\}=1 \text {. }
$$


Proof. Let $\zeta_{t}=X_{t}(p)-X_{t}(q)$ for $t \geq 0$. From (4.1) we have

$$
\begin{aligned}
& \zeta_{t}= \zeta_{0} \\
&+\int_{0}^{t} \int_{\mathbb{N}_{+}} \int_{[0, p]} \int_{X_{s-}(q)}^{X_{s-}(p)}(z-1) N(\mathrm{~d} s, \mathrm{~d} z, \mathrm{~d} \theta, \mathrm{d} u) \\
&-\int_{0}^{t} \int_{\mathbb{N}_{+}} \int_{(p, q]} \int_{0}^{X_{s-}(q)}(z-1) N(\mathrm{~d} s, \mathrm{~d} z, \mathrm{~d} \theta, \mathrm{d} u) \\
&-\int_{0}^{t} \int_{0}^{b(q)} \int_{X_{s-}(q)}^{X_{s-}(p)} N_{0}(\mathrm{~d} s, \mathrm{~d} \theta, \mathrm{d} u)-\int_{0}^{t} \int_{b(q)}^{b(p)} \int_{0}^{X_{s-}(p)} N_{0}(\mathrm{~d} s, \mathrm{~d} \theta, \mathrm{d} u) .
\end{aligned}
$$

Let $\tau_{m}=\inf \left\{t \geq 0: X_{t}(q) \geq m\right.$ or $\left.X_{t}(p) \geq m\right\}$. It is easy to construct a sequence of functions $\left\{f_{n}\right\}$ on $\mathbb{R}$ such that $0 \leq f_{n}^{\prime}(z) \leq 1$ for $z \geq 0$, and $f_{n}(z)=f_{n}^{\prime}(z)=0$ for $z \leq 0$. Moreover, $f_{n}(z) \rightarrow z^{+}:=0 \vee z$ increasingly as $n \rightarrow \infty$. By (4.2) and Itô's formula,

$$
\begin{aligned}
f_{n}\left(\zeta_{t \wedge \tau_{m}}\right)= & \int_{0}^{t \wedge \tau_{m}} \int_{\mathbb{N}_{+}} \int_{[0, p]} \int_{X_{s-}(q)}^{X_{s-}(p)}\left[f_{n}\left(\zeta_{s-}+z-1\right)-f_{n}\left(\zeta_{s-}\right)\right] \\
& +\int_{0}^{t \wedge \tau_{m}} \int_{\mathbb{N}_{+}} \int_{(p, q]} \int_{0}^{X_{s-}(q)}\left[f_{n}\left(\zeta_{s-}-z+1\right)-f_{n}\left(\zeta_{s-}\right)\right] N(\mathrm{~d} s, \mathrm{~d} z, \mathrm{~d} \theta, \mathrm{d} \theta, \mathrm{d} u) \\
& +\int_{0}^{t \wedge \tau_{m}} \int_{0}^{b(q)} \int_{X_{s-}(p)}^{X_{s-}(q)}\left[f_{n}\left(\zeta_{s-}-1\right)-f_{n}\left(\zeta_{s-}\right)\right] \mathbf{1}_{\left\{\zeta_{s-}>0\right\}} N_{0}(\mathrm{~d} s, \mathrm{~d} \theta, \mathrm{d} u) \\
& +\int_{0}^{t \wedge \tau_{m}} \int_{b(q)}^{b(p)} \int_{0}^{X_{s-}(p)}\left[f_{n}\left(\zeta_{s-}-1\right)-f_{n}\left(\zeta_{s-}\right)\right] N_{0}(\mathrm{~d} s, \mathrm{~d} \theta, \mathrm{d} u) \\
\leq & \sigma \int_{0}^{t \wedge \tau_{m}} \zeta_{s-} \mathbf{1}_{\left\{\zeta_{s-}>0\right\}} \mathrm{d} s \int_{\mathbb{N}_{+}}(z-1) \pi_{p}(\mathrm{~d} z)+\text { martingale. }
\end{aligned}
$$

Taking the expectation in both sides and letting $n \rightarrow \infty$ gives

$$
\mathbb{E}\left[\zeta_{t \wedge \tau_{m}}^{+}\right] \leq \sigma\left(g_{p}^{\prime}(1)-1+b(p)\right) \int_{0}^{t} \mathbb{E}\left[\zeta_{s \wedge \tau_{m}}^{+}\right] \mathrm{d} s .
$$

Hence, $\mathbb{E}\left[\zeta_{t \wedge \tau_{m}}^{+}\right]=0$ for all $t \geq 0$. Since $\tau_{m} \rightarrow \infty$ as $m \rightarrow \infty$, this proves the desired comparison result.

Proposition 4.1. There is a locally bounded positive function $(t, u) \mapsto C(t, u)$ on $[0, \infty)^{2}$ satisfying, for any $t \geq 0$ and $p \leq q \leq u<\infty$,

$$
\mathbb{E}\left\{\sup _{0 \leq s \leq t}\left[X_{s}(q)-X_{s}(p)\right]\right\} \leq C(t, u)\left\{X_{0}(q)-X_{0}(p)+g_{q}^{\prime}(1)-g_{p}^{\prime}(1)\right\} .
$$

Proof. Let $\xi_{t}=X_{t}(q)-X_{t}(p)$. From (4.1) we obtain

$$
\begin{aligned}
& \sup _{0 \leq s \leq t} \xi_{s} \leq \xi_{0} \\
&+\int_{0}^{t} \int_{\mathbb{N}_{+}} \int_{[0, q]} \int_{X_{s-}(p)}^{X_{s-}(q)}(z-1) N(\mathrm{~d} s, \mathrm{~d} z, \mathrm{~d} \theta, \mathrm{d} u) \\
&+\int_{0}^{t} \int_{\mathbb{N}_{+}} \int_{(p, q]} \int_{0}^{X_{s-}(p)}(z-1) N(\mathrm{~d} s, \mathrm{~d} z, \mathrm{~d} \theta, \mathrm{d} u) \\
&+\int_{0}^{t} \int_{b(q)}^{b(p)} \int_{0}^{X_{s-}(p)} N_{0}(\mathrm{~d} s, \mathrm{~d} \theta, \mathrm{d} u) .
\end{aligned}
$$


Hence,

$$
\mathbb{E}\left[\sup _{0 \leq s \leq t} \xi_{s}\right] \leq \xi_{0}+\sigma\left[g_{p}^{\prime}(1)-1+b(q)\right] \int_{0}^{t} \mathbb{E}\left[\xi_{s}\right] \mathrm{d} s+\sigma\left[g_{q}^{\prime}(1)-g_{p}^{\prime}(1)\right] \int_{0}^{t} \mathbb{E}\left[X_{s}(p)\right] \mathrm{d} s .
$$

Since $t \mapsto \mathbb{E}\left[X_{t}(p)\right]$ is locally bounded, by Gronwall's inequality, we obtain the desired estimate, completing the proof.

From the discussion above, given a constant $\sigma>0$ and a family of probability generating functions $\left\{g_{\theta}: \theta \geq 0\right\}$, we obtain a continuous-time and discrete-state branching process flow $\left\{X_{t}(q): t \geq 0, q \geq 0\right\}$ as the solution of equation (4.1). For every $t \geq 0$, define the random function $\tilde{X}_{t} \in F[0,1]$ by $\tilde{X}_{t}(1)=X_{t}(1)$ and

$$
\tilde{X}_{t}(q)=\inf \left\{X_{t}(u): \text { rational } u \in(q, 1]\right\}, \quad 0 \leq q<1 .
$$

By Proposition 4.1, for each $q \in[0,1]$, we have

$$
\mathbb{P}\left\{\tilde{X}_{t}(q)=X_{t}(q) \text { for all } t \geq 0\right\}=1 .
$$

Therefore, $\left\{\tilde{X}_{t}(q): t \geq 0\right\}$ is also càdlàg and solves (4.1) for every $q \in[0,1]$.

\section{Scaling limits of the discrete branching flows}

In this section we prove some limit theorems for the discrete-state branching flows, which will lead to the continuous-state branching flows of Li (2014). We shall present the limit theorems in the settings of measure-valued processes and path-valued processes.

Suppose that, for each $k \geq 1$, there is a positive constant $\sigma_{k}$ and a family of generating functions $\left\{g_{\theta}^{(k)}: \theta \geq 0\right\}$ satisfying the assumptions specified at the beginning of Section 4 . Then we can define $\pi_{\theta}^{(k)}(\mathrm{d} z)$ and $\bar{\pi}^{(k)}(\mathrm{d} z, \mathrm{~d} \theta)$ in the same way as there. Let $\left\{X_{t}^{(k)}(q): t \geq 0\right\}$ be the corresponding solution of (4.1) and $\left\{\tilde{X}_{t}^{(k)}(q): t \geq 0, q \in[0, k]\right\}$ be defined in the same way as in (4.3). Define

$$
Y_{t}^{(k)}(q)=\frac{1}{k} \tilde{X}_{t}^{(k)}(k q), \quad q \in[0,1]
$$

From (4.1) we have

$$
\begin{aligned}
Y_{t}^{(k)}(q)= & Y_{0}^{(k)}(q)+\frac{1}{k} \int_{0}^{t} \int_{\mathbb{N}_{+}} \int_{[0, k q]} \int_{0}^{k Y_{s-}^{(k)}(q)}(z-1) N(\mathrm{~d} s, \mathrm{~d} z, \mathrm{~d} \theta, \mathrm{d} u) \\
& -\frac{1}{k} \int_{0}^{t} \int_{0}^{b_{k}(k q)} \int_{0}^{k Y_{s-}^{(k)}(q)} N_{0}(\mathrm{~d} s, \mathrm{~d} \theta, \mathrm{d} u) .
\end{aligned}
$$

We can use a standard stopping time argument to show that, for any $q \in[0,1]$, the function $t \mapsto \mathbb{E}\left[Y_{t}^{(k)}(q)\right]$ is locally bounded. Then, by an argument similar to the proof of Proposition 3.2, we have the following proposition.

Proposition 5.1. For any $t \geq 0$ and $q \in[0,1]$, we have

$$
\mathbb{E}\left[\sup _{0 \leq s \leq t} Y_{s}^{(k)}(q)\right] \leq Y_{0}^{(k)}(q) \exp \left\{t \sigma_{k}\left(\left(g_{k q}^{(k)}\right)^{\prime}(1)-1+b_{k}(k q)\right)\right\}
$$

The random function $Y_{t}^{(k)} \in F[0,1]$ induces a random measure $Y_{t}^{(k)} \in M[0,1]$ so that $Y_{t}^{(k)}([0, q])=Y_{t}^{(k)}(q)$ for $q \in[0,1]$. We are interested in the asymptotic behavior of the 
process $\left\{Y_{t}^{(k)}: t \geq 0\right\}$ as $k \rightarrow \infty$. For any $f \in C^{1}[0,1]$, we can use Fubini's theorem to see that

$$
\left\langle Y_{t}^{(k)}, f\right\rangle=f(1) Y_{t}^{(k)}(1)-\int_{0}^{1} f^{\prime}(q) Y_{t}^{(k)}(q) \mathrm{d} q .
$$

Fix an integer $n \geq 1$ and let $q_{i}=i / 2^{n}$ for $i=0,1, \ldots, 2^{n}$. By (5.1), we have

$$
\begin{aligned}
\sum_{i=1}^{2^{n}} f^{\prime}\left(q_{i}\right) Y_{t}^{(k)}\left(q_{i}\right)= & \sum_{i=1}^{2^{n}} f^{\prime}\left(q_{i}\right) Y_{0}^{(k)}\left(q_{i}\right) \\
& +\frac{1}{k} \sum_{i=1}^{2^{n}} f\left(q_{i}\right) \int_{0}^{t} \int_{\mathbb{N}_{+}} \int_{\left[0, k q_{i}\right]} \int_{0}^{k Y_{s-}^{(k)}\left(q_{i}\right)}(z-1) N(\mathrm{~d} s, \mathrm{~d} z, \mathrm{~d} \theta, \mathrm{d} u) \\
& -\frac{1}{k} \sum_{i=1}^{2^{n}} f^{\prime}\left(q_{i}\right) \int_{0}^{t} \int_{0}^{b k_{k}\left(k q_{i}\right)} \int_{0}^{k Y_{s-}^{(k)}\left(q_{i}\right)} N_{0}(\mathrm{~d} s, \mathrm{~d} \theta, \mathrm{d} u) \\
= & \sum_{i=1}^{2^{n}} f^{\prime}\left(q_{i}\right) Y_{0}^{(k)}\left(q_{i}\right) \\
& +\frac{1}{k} \int_{0}^{t} \int_{\mathbb{N}_{+}} \int_{[0, k]} \int_{0}^{k Y_{s-}^{(k)}(1)} F_{n}^{(k)}(s, \theta, u)(z-1) N(\mathrm{~d} s, \mathrm{~d} z, \mathrm{~d} \theta, \mathrm{d} u) \\
& -\frac{1}{k} \int_{0}^{t} \int_{0}^{b_{k}(0)} \int_{0}^{k Y_{s-}^{(k)}(1)} \tilde{F}_{n}^{(k)}(s, \theta, u) N_{0}(\mathrm{~d} s, \mathrm{~d} \theta, \mathrm{d} u),
\end{aligned}
$$

where

$$
\begin{gathered}
F_{n}^{(k)}(s, \theta, u)=\sum_{i=1}^{2^{n}} f^{\prime}\left(q_{i}\right) \mathbf{1}_{\left\{\theta \leq k q_{i}\right\}} \mathbf{1}_{\left\{u \leq k Y_{s-}^{(k)}\left(q_{i}\right)\right\}} \\
\text { and } \tilde{F}_{n}^{(k)}(s, \theta, u)=\sum_{i=1}^{2^{n}} f^{\prime}\left(q_{i}\right) \mathbf{1}_{\left\{\theta \leq b_{k}\left(k q_{i}\right)\right\}} \mathbf{1}_{\left\{u \leq k Y_{s-}^{(k)}\left(q_{i}\right)\right\}} .
\end{gathered}
$$

By the right continuity of $q \mapsto Y_{t}^{(k)}(q)$, it is easy to see that, as $n \rightarrow \infty$,

$$
2^{-n} F_{n}^{(k)}(s, \theta, u) \rightarrow F^{(k)}(s, \theta, u):=\int_{0}^{1} f^{\prime}(q) \mathbf{1}_{\{\theta \leq k q\}} \mathbf{1}_{\left\{u \leq k Y_{s-}^{(k)}(q)\right\}} \mathrm{d} q
$$

and

$$
2^{-n} \tilde{F}_{n}^{(k)}(s, \theta, u) \rightarrow \tilde{F}^{(k)}(s, \theta, u):=\int_{0}^{1} f^{\prime}(q) \mathbf{1}_{\left\{\theta \leq b_{k}(k q)\right\}} \mathbf{1}_{\left\{u \leq k Y_{s-}^{(k)}(q)\right\}} \mathrm{d} q .
$$

Then, by (5.3), we have, almost surely,

$$
\begin{aligned}
\int_{0}^{1} f^{\prime}(q) Y_{t}^{(k)}(q) \mathrm{d} q= & \int_{0}^{1} f^{\prime}(q) Y_{0}^{(k)}(q) \mathrm{d} q \\
& +\frac{1}{k} \int_{0}^{t} \int_{\mathbb{N}_{+}} \int_{[0, k]} \int_{0}^{k Y_{s-}^{(k)}(1)} F^{(k)}(s, \theta, u)(z-1) N(\mathrm{~d} s, \mathrm{~d} z, \mathrm{~d} \theta, \mathrm{d} u) \\
& -\frac{1}{k} \int_{0}^{t} \int_{0}^{b_{k}(0)} \int_{0}^{k Y_{s-}^{(k)}(1)} \tilde{F}^{(k)}(s, \theta, u) N_{0}(\mathrm{~d} s, \mathrm{~d} \theta, \mathrm{d} u)
\end{aligned}
$$


From (5.1), (5.2), and (5.4), it follows that, almost surely,

$$
\begin{aligned}
\left\langle Y_{t}^{(k)}, f\right\rangle= & \left\langle Y_{0}^{(k)}, f\right\rangle \\
& +\frac{1}{k} \int_{0}^{t} \int_{\mathbb{N}_{+}} \int_{[0, k]} \int_{0}^{k Y_{s-}^{(k)}(1)}\left[f(1)-F^{(k)}(s, \theta, u)\right](z-1) N(\mathrm{~d} s, \mathrm{~d} z, \mathrm{~d} \theta, \mathrm{d} u) \\
& -\frac{1}{k} \int_{0}^{t} \int_{0}^{b_{k}(k)} \int_{0}^{k Y_{s-}^{(k)}(1)}\left[f(1)-\tilde{F}^{(k)}(s, \theta, u)\right] N_{0}(\mathrm{~d} s, \mathrm{~d} \theta, \mathrm{d} u) \\
& +\frac{1}{k} \int_{0}^{t} \int_{b_{k}(k)}^{b_{k}(0)} \int_{0}^{k Y_{s-}^{(k)}(1)} \tilde{F}^{(k)}(s, \theta, u) N_{0}(\mathrm{~d} s, \mathrm{~d} \theta, \mathrm{d} u) .
\end{aligned}
$$

Proposition 5.2. Suppose that $Y_{0}^{(k)}(1)$ converges to some $Y_{0}(1)$ as $k \rightarrow \infty$ and

$$
\sup _{k \geq 1} \sigma_{k}\left[\left(g_{k}^{(k)}\right)^{\prime}(1)-1+b_{k}(0)\right]<\infty .
$$

Then $\left\{Y_{t}^{(k)}: t \geq 0\right\}, k=1,2, \ldots$ is a tight sequence in $D([0, \infty), M[0,1])$.

Proof. For any $t \geq 0$ and $f \in C[0,1]$, by Proposition 5.1, it is easy to see that

$$
t \mapsto C_{t}:=\sup _{k \geq 1} \mathbb{E}\left[\sup _{0 \leq s \leq t}\left\langle Y_{s}^{(k)}, f\right\rangle\right]
$$

is locally bounded. Hence, for every fixed $t \geq 0$, the sequence $\left\langle Y_{t}^{(k)}, f\right\rangle$ is tight. Let $\tau_{k}$ be a bounded stopping time for $\left\{Y_{t}^{(k)}: t \geq 0\right\}$ and assume that the sequence $\left\{\tau_{k}: k=1,2, \ldots\right\}$ is bounded above by $T \geq 0$. Let $f \in C^{1}[0,1]$. By (5.5), we see that

$$
\begin{aligned}
& \mathbb{E}\left[\left|\left\langle Y_{\tau_{k}+t}^{(k)}, f\right\rangle-\left\langle Y_{\tau_{k}}^{(k)}, f\right\rangle\right|\right] \\
& \leq \frac{\sigma_{k}}{k} \mathbb{E}\left[\int_{0}^{t} \mathrm{~d} s \int_{\mathbb{N}_{+}} \int_{[0, k]} \int_{0}^{k Y_{s+\tau_{k}}^{(k)}(1)}(z-1)\left|f(1)-F^{(k)}\left(s+\tau_{k}, \theta, u\right)\right| \bar{\pi}^{(k)}(\mathrm{d} z, \mathrm{~d} \theta) \mathrm{d} u\right] \\
& \quad+\frac{\sigma_{k}}{k} \mathbb{E}\left[\int_{0}^{t} \mathrm{~d} s \int_{0}^{b_{k}(k)} \mathrm{d} \theta \int_{0}^{k Y_{s+\tau_{k}}^{(k)}(1)}\left|f(1)-\tilde{F}^{(k)}\left(s+\tau_{k}, \theta, u\right)\right| \mathrm{d} u\right] \\
& \quad+\frac{\sigma_{k}}{k} \mathbb{E}\left[\int_{0}^{t} \mathrm{~d} s \int_{b_{k}(k)}^{b_{k}(0)} \mathrm{d} \theta \int_{0}^{k Y_{s+\tau_{k}}^{(k)}(1)}\left|\tilde{F}^{(k)}\left(s+\tau_{k}, \theta, u\right)\right| \mathrm{d} u\right] .
\end{aligned}
$$

For $s, \theta, u>0$, let $Y_{s, k}^{-1}(u)=\inf \left\{q \geq 0: Y_{s}^{(k)}(q)>u\right\}$ and $b_{k}^{-1}(u)=\inf \left\{q \geq 0: b_{k}(q)>u\right\}$. It is easy to see that

$$
\left\{q \geq 0: u \leq k Y_{s}^{(k)}(q)\right\}=\left[Y_{s, k}^{-1}(u / k), \infty\right), \quad\left\{q \geq 0: \theta \leq b_{k}(k q)\right\}=\left[0, b_{k}^{-1}(\theta) / k\right]
$$

except for at most countably many $u>0$ and $\theta>0$, respectively. Hence, in (5.6) we can replace $f(1)-F^{(k)}(s, \theta, u)$ by

$$
f(1)-\int_{\theta / k}^{1} f^{\prime}(q) \mathbf{1}_{\left\{Y_{s, k}^{-1}(u / k) \leq q\right\}} \mathrm{d} q=f\left(Y_{s, k}^{-1}\left(\frac{u}{k}\right) \vee \frac{\theta}{k}\right)
$$

and $\tilde{F}^{(k)}(s, \theta, u)$ can be replaced by

$$
\begin{aligned}
& \int_{0}^{1} f^{\prime}(q) \mathbf{1}_{\left\{q \leq b_{k}^{-1}(\theta) / k\right\}} \mathbf{1}_{\left\{Y_{s, k}^{-1}(u / k) \leq q\right\}} \mathrm{d} q \\
& \quad=\left[f\left(1 \wedge\left(b_{k}^{-1}(\theta) / k\right)\right)-f\left(Y_{s, k}^{-1}(u / k)\right)\right] \mathbf{1}_{\left\{Y_{s, k}^{-1}(u / k) \leq b_{k}^{-1}(\theta) / k\right\}} .
\end{aligned}
$$


Then, from (5.6), we have

$$
\begin{aligned}
& \mathbb{E}\left[\left|\left\langle Y_{\tau_{k}+t}^{(k)}, f\right\rangle-\left\langle Y_{\tau_{k}}^{(k)}, f\right\rangle\right|\right] \\
& \leq \sigma_{k} \mathbb{E}\left[\int_{0}^{t} \mathrm{~d} s \int_{0}^{1} Y_{s+\tau_{k}}^{(k)}(\mathrm{d} x) \int_{\mathbb{N}_{+}} \int_{[0, k]}(z-1)|f(x \vee \theta)| \bar{\pi}^{(k)}(\mathrm{d} z, \mathrm{~d} \theta)\right] \\
&+\sigma_{k} \mathbb{E}\left[\int_{0}^{t} \mathrm{~d} s \int_{0}^{b_{k}(k)} \mathrm{d} \theta \int_{0}^{1}|f(x)| Y_{s+\tau_{k}}^{(k)}(\mathrm{d} x)\right] \\
&+\sigma_{k} \mathbb{E}\left[\int_{0}^{t} \mathrm{~d} s \int_{b_{k}(k)}^{b_{k}(0)} \mathrm{d} \theta \int_{0}^{1}\left|f\left(\frac{b_{k}^{-1}(\theta)}{k}\right)-f(x)\right| Y_{s+\tau_{k}}^{(k)}(\mathrm{d} x)\right] \\
& \leq\|f\| \sigma_{k} \int_{0}^{t} \mathbb{E}\left[Y_{s+\tau_{k}}^{(k)}(1)\right] \mathrm{d} s \int_{\mathbb{N}_{+}}(z-1) \pi_{k}^{(k)}(\mathrm{d} z) \\
&+\|f\| \sigma_{k} b_{k}(k) \mathbb{E} \int_{0}^{t}\left[Y_{s+\tau_{k}}^{(k)}(1)\right] \mathrm{d} s \\
&+2\|f\| \sigma_{k}\left[b_{k}(0)-b_{k}(k)\right] \int_{0}^{t} \mathbb{E}\left[Y_{s+\tau_{k}}^{(k)}(1)\right] \mathrm{d} s \\
& \leq\|f\| \sigma_{k}\left(\left(g_{k}^{(k)}\right)^{\prime}(1)-1+2 b_{k}(0)\right) \int_{0}^{t} \mathbb{E}\left[Y_{s+\tau_{k}}^{(k)}(1)\right] \mathrm{d} s \\
& \leq 2\|f\| Y_{0}^{(k)}(1) t \sigma_{k} A_{k} \exp \left\{\sigma_{k} A_{k}(t+T)\right\},
\end{aligned}
$$

where $A_{k}=\left(g_{k}^{(k)}\right)^{\prime}(1)-1+b_{k}(0)$ and the last inequality follows by Proposition 5.1. For $f \in C[0,1]$, the above inequality follows by an approximation argument. From (5.7) we have

$$
\lim _{t \rightarrow 0} \sup _{k \geq 1} \mathbb{E}\left[\left|\left\langle Y_{\tau_{k}+t}^{(k)}, f\right\rangle-\left\langle Y_{\tau_{k}}^{(k)}, f\right\rangle\right|\right]=0 .
$$

By a criterion of Aldous (1978), the sequence $\left\{\left\langle Y_{t}^{(k)}, f\right\rangle: t \geq 0\right\}$ is tight in $D([0, \infty), \mathbb{R})$; see also Ethier and Kurtz (1986, pp. 137-138). Hence, the tightness criterion of Roelly (1986) implies that $\left\{Y_{t}^{(k)}: t \geq 0\right\}$ is tight in $D([0, \infty), M[0,1])$.

For $z \geq 0$, define

$$
\phi_{\theta}^{(k)}(z)=k \sigma_{k}\left[g_{k \theta}^{(k)}\left(\mathrm{e}^{-z / k}\right)-\mathrm{e}^{-z / k}\right] .
$$

Let us consider the following assumption.

Assumption 5.1. For each $l \geq 0$, the sequence $\left\{\phi_{\theta}^{(k)}(z)\right\}$ is Lipschitz with respect to $z$ uniformly on $[0,1] \times[0, l]$ and there is an admissible family of branching mechanisms $\left\{\phi_{\theta}(z): \theta \geq 0\right\}$ with $(\partial / \partial \theta) \phi_{\theta}(z)=-\psi_{\theta}(z)$ such that $\phi_{\theta}^{(k)}(z) \rightarrow \phi_{\theta}(z)$ uniformly on $[0,1] \times[0, l]$ as $k \rightarrow \infty$.

Let $\left\{Y_{t}: t \geq 0\right\}$ be the càdlàg superprocess with the transition semigroup defined by (2.3) and (2.4).

Theorem 5.1. Suppose that Assumption 5.1 holds and $\sup _{k \geq 1} \sigma_{k} b_{k}(0)<\infty$. If $Y_{0}^{(k)}$ converges weakly to $Y_{0} \in M[0,1]$ then $\left\{Y_{t}^{(k)}: t \geq 0\right\}$ converges in distribution to the superprocess $\left\{Y_{t}: t \geq 0\right\}$ on $D([0, \infty), M[0,1])$.

Proof. Under the assumption, we have

$$
\sup _{k \geq 1} \sigma_{k}\left[\left(g_{k}^{(k)}\right)^{\prime}(1)-1+b_{k}(0)\right]<\infty .
$$


By Proposition 5.2 and Skorokhod's representation theorem, to simplify the notation we pass to a subsequence and simply assume $\left\{Y_{t}^{(k)}: t \geq 0\right\}$ converges a.s. to a process $\left\{Z_{t}: t \geq 0\right\}$ in the topology of $D([0, \infty), M[0,1])$. Since the solution of the martingale problem (2.2) is unique, it suffices to prove that the weak limit point $\left\{Z_{t}: t \geq 0\right\}$ of the sequence $\left\{Y_{t}^{(k)}: t \geq 0\right\}$ is the solution of the martingale problem. Let $Y_{s, k}^{-1}(u)$ and $b_{k}^{-1}(u)$ be defined as in Proposition 5.2. For every $G \in C^{2}(\mathbb{R})$ and $f \in C^{1}[0,1]$, we use (5.5) and Itô's formula to obtain

$$
\begin{aligned}
& G\left(\left\langle Y_{t}^{(k)}, f\right\rangle\right) \\
& =G\left(\left\langle Y_{0}^{(k)}, f\right\rangle\right) \\
& +\sigma_{k} \int_{0}^{t} \mathrm{~d} s \int_{\mathbb{N}_{+}} \int_{[0, k]} \int_{0}^{k Y_{s-}^{(k)}(1)}\left\{G\left(\left\langle Y_{s-}^{(k)}, f\right\rangle+k^{-1}(z-1)\left[f(1)-F^{(k)}(s, \theta, u)\right]\right)\right. \\
& \left.-G\left(\left\langle Y_{s-}^{(k)}, f\right\rangle\right)\right\} \bar{\pi}^{(k)}(\mathrm{d} z, \mathrm{~d} \theta) \mathrm{d} u \\
& +\sigma_{k} \int_{0}^{t} \mathrm{~d} s \int_{0}^{b_{k}(k)} \mathrm{d} \theta \int_{0}^{k Y_{s-}^{(k)}(1)}\left\{G\left(\left\langle Y_{s-}^{(k)}, f\right\rangle-k^{-1}\left[f(1)-\tilde{F}^{(k)}(s, \theta, u)\right]\right)\right. \\
& \left.-G\left(\left\langle Y_{s-}^{(k)}, f\right\rangle\right)\right\} \mathrm{d} u \\
& +\sigma_{k} \int_{0}^{t} \mathrm{~d} s \int_{b_{k}(k)}^{b_{k}(0)} \mathrm{d} \theta \int_{0}^{k Y_{s-}^{(k)}(1)}\left\{G\left(\left\langle Y_{s-}^{(k)}, f\right\rangle+k^{-1} \tilde{F}^{(k)}(s, \theta, u)\right)-G\left(\left\langle Y_{s-}^{(k)}, f\right\rangle\right)\right\} \mathrm{d} u \\
& + \text { local martingale } \\
& =G\left(\left\langle Y_{0}^{(k)}, f\right\rangle\right) \\
& +\sigma_{k} \int_{0}^{t} \mathrm{~d} s \int_{\mathbb{N}_{+}} \int_{[0, k]} \int_{0}^{k Y_{s-}^{(k)}(1)}\left\{G\left(\left\langle Y_{s-}^{(k)}, f\right\rangle+k^{-1}(z-1) f\left(Y_{s, k}^{-1}(u / k) \vee(\theta / k)\right)\right)\right. \\
& \left.-G\left(\left\langle Y_{s-}^{(k)}, f\right\rangle\right)\right\} \bar{\pi}^{(k)}(\mathrm{d} z, \mathrm{~d} \theta) \mathrm{d} u \\
& +\sigma_{k} \int_{0}^{t} \mathrm{~d} s \int_{0}^{b_{k}(k)} \mathrm{d} \theta \int_{0}^{k Y_{s-}^{(k)}(1)}\left\{G\left(\left\langle Y_{s-}^{(k)}, f\right\rangle-k^{-1} f\left(Y_{s, k}^{-1}(u / k)\right)\right)\right. \\
& \left.-G\left(\left\langle Y_{s-}^{(k)}, f\right\rangle\right)\right\} \mathrm{d} u \\
& +\sigma_{k} \int_{0}^{t} \mathrm{~d} s \int_{b_{k}(k)}^{b_{k}(0)} \mathrm{d} \theta \int_{0}^{k Y_{s-}^{(k)}(1)}\left\{G \left(\left\langle Y_{s-}^{(k)}, f\right\rangle+k^{-1}\left[f\left(b_{k}^{-1}(\theta) / k\right)-f\left(Y_{s, k}^{-1}(u / k)\right)\right]\right.\right. \\
& \left.\times \mathbf{1}_{\left\{Y_{s, k}^{-1}(u / k) \leq b_{k}^{-1}(\theta) / k\right\}}\right) \\
& \left.-G\left(\left\langle Y_{s-}^{(k)}, f\right\rangle\right)\right\} \mathrm{d} u+\text { local martingale } \\
& =G\left(\left\langle Y_{0}^{(k)}, f\right\rangle\right) \\
& +k \sigma_{k} \int_{0}^{t} \mathrm{~d} s \int_{[0,1]} Y_{s-}^{(k)}(\mathrm{d} x) \int_{\mathbb{N}_{+}} \int_{[0,1]}\left\{G\left(\left\langle Y_{s-}^{(k)}, f\right\rangle+k^{-1}(z-1) f(x \vee \theta)\right)\right. \\
& \left.-G\left(\left\langle Y_{s-}^{(k)}, f\right\rangle\right)\right\} \bar{\pi}^{(k)}(\mathrm{d} z, k \mathrm{~d} \theta) \\
& +k \sigma_{k} b_{k}(k) \int_{0}^{t} \mathrm{~d} s \int_{[0,1]}\left\{G\left(\left\langle Y_{s-}^{(k)}, f\right\rangle-k^{-1} f(x)\right)-G\left(\left\langle Y_{s-}^{(k)}, f\right\rangle\right)\right\} Y_{s-}^{(k)}(\mathrm{d} x) \\
& +k \sigma_{k} \int_{0}^{t} \mathrm{~d} s \int_{[0,1]} Y_{s-}^{(k)}(\mathrm{d} x) \int_{1}^{x}\left\{G\left(\left\langle Y_{s-}^{(k)}, f\right\rangle+k^{-1}[f(\theta)-f(x)]\right)\right. \\
& \left.-G\left(\left\langle Y_{s-}^{(k)}, f\right\rangle\right)\right\} b_{k}(k \mathrm{~d} \theta)+\text { local martingale }
\end{aligned}
$$




$$
\begin{aligned}
= & G\left(\left\langle Y_{0}^{(k)}, f\right\rangle\right) \\
& +k \sigma_{k} \int_{0}^{t} \mathrm{~d} s \int_{[0,1]} Y_{s-}^{(k)}(\mathrm{d} x) \int_{\mathbb{N}} \int_{[0,1]}\left\{G\left(\left\langle Y_{s-}^{(k)}, f\right\rangle+k^{-1}(z-1) f(x \vee \theta)\right)\right. \\
& +k \sigma_{k} \int_{0}^{t} \mathrm{~d} s \int_{[0,1]} Y_{s-}^{(k)}(\mathrm{d} x) \int_{\{0\}} \int_{x}^{1} \varepsilon_{k}(s, x, \theta) \bar{\pi}^{(k)}(\mathrm{d} z, k \mathrm{~d} \theta) \\
& + \text { local martingale, }
\end{aligned}
$$

where

$$
\begin{aligned}
\varepsilon_{k}(s, \theta, x)= & \left\{G\left(\left\langle Y_{s-}^{(k)}, f\right\rangle-k^{-1} f(x)\right)-G\left(\left\langle Y_{s-}^{(k)}, f\right\rangle-k^{-1} f(\theta)\right)\right\} \\
& -\left\{G\left(\left\langle Y_{s-}^{(k)}, f\right\rangle+k^{-1}[f(\theta)-f(x)]\right)-G\left(\left\langle Y_{s-}^{(k)}, f\right\rangle\right)\right\} .
\end{aligned}
$$

It is easy to see that

$$
k \sigma_{k} \int_{\{0\}} \int_{x}^{1} \varepsilon_{k}(s, x, \theta) \bar{\pi}^{(k)}(\mathrm{d} z, k \mathrm{~d} \theta)
$$

tends to 0 uniformly as $k \rightarrow \infty$. Let $G(x)=\mathrm{e}^{-x}$. By letting $k \rightarrow \infty$ in (5.8) we obtain (2.2) for $f \in C^{1}[0,1]$. A simple approximation shows that the martingale problem (2.2) actually holds for all $f \in C[0,1]$. By the proof of Theorem 7.13 in Li (2011), we obtain the result completing the proof.

Let $\left\{0 \leq a_{1}<a_{2}<\cdots<a_{n}=1\right\}$ be an ordered set of constants. Denote by $\left\{Y_{t, a_{i}}: t \geq 0\right\}$ and $\left\{Y_{t, a_{i}}^{(k)}: t \geq 0\right\}$ the restriction of $\left\{Y_{t}: t \geq 0\right\}$ and $\left\{Y_{t}^{(k)}: t \geq 0\right\}$ to $\left[0, a_{i}\right]$, respectively. Let $Y_{t}\left(a_{i}\right):=Y_{t}\left[0, a_{i}\right]$ and $Y_{t}^{(k)}\left(a_{i}\right):=Y_{t}^{(k)}\left[0, a_{i}\right]$ for every $t \geq 0, i=1,2, \ldots, n$. By arguments similar to those in $\mathrm{He}$ and $\mathrm{Ma}$ (2014), we have the following results.

Theorem 5.2. Suppose that Assumption 5.1 is satisfied and $\sup _{k \geq 1} \sigma_{k} b_{k}(0)<\infty$. If $Y_{0}^{(k)}$ converges weakly to $Y_{0} \in M[0,1]$ then $\left\{\left(Y_{t, a_{1}}^{(k)}, \ldots, Y_{t, a_{n}}^{(k)}\right): t \geq 0\right\}$ converges in distribution to $\left\{\left(Y_{t, a_{1}}, \ldots, Y_{t, a_{n}}\right): t \geq 0\right\}$ on $D\left([0, \infty), M\left[0, a_{1}\right] \times \cdots \times M\left[0, a_{n}\right]\right)$.

Corollary 5.1. Suppose that Assumption 5.1 is satisfied and $\sup _{k \geq 1} \sigma_{k} b_{k}(0)<\infty$. If

$$
\left(Y_{0}^{(k)}\left(a_{1}\right), \ldots, Y_{0}^{(k)}\left(a_{n}\right)\right)
$$

converges to $\left(Y_{0}\left(a_{1}\right), \ldots, Y_{0}\left(a_{n}\right)\right)$ then it follows that $\left\{\left(Y_{t}^{(k)}\left(a_{1}\right), \ldots, Y_{t}^{(k)}\left(a_{n}\right)\right): t \geq 0\right\}$ converges in distribution to $\left\{\left(Y_{t}\left(a_{1}\right), \ldots, Y_{t}\left(a_{n}\right)\right): t \geq 0\right\}$ on $D\left([0, \infty), \mathbb{R}_{+}^{n}\right)$.

Example 5.1. Suppose that $\phi$ is defined in (2.1). Let $\Theta_{\phi}$ be the set of $\theta \geq 0$ such that

$$
\int_{1}^{\infty} u \mathrm{e}^{\theta u} m(\mathrm{~d} u)<\infty .
$$

Then a particular choice of $\phi_{\theta}$ is

$$
\phi_{\theta}(\cdot)=\phi(\cdot-\theta)-\phi(-\theta), \quad \theta \in \Theta_{\phi} .
$$

Suppose that $[0,1] \subset \Theta_{\phi}$. Since $\phi_{1}(\cdot)$ is a branching mechanism, Li (2011, p.93) implies that there is a sequence of generating functions $\left\{g_{k}^{(k)}: k \geq 1\right\}$ and some $\sigma_{k}$ such that

$$
k \sigma_{k}\left[g_{k}^{(k)}\left(\mathrm{e}^{-z / k}\right)-\mathrm{e}^{-z / k}\right] \rightarrow \phi(z-1)-\phi(-1) \quad \text { as } k \rightarrow \infty .
$$


Hence, for $\theta \in[0,1]$, define

$$
g_{k \theta}^{(k)}(z)=1-\mathrm{e}^{(1-\theta) / k} g_{k}^{(k)}\left(\mathrm{e}^{-(1-\theta) / k}\right)+\mathrm{e}^{(1-\theta) / k} g_{k}^{(k)}\left(\mathrm{e}^{-(1-\theta) / k} z\right)
$$

The reader can check that Assumption 5.1 holds with $\phi_{\theta}(\cdot)=\phi(\cdot-\theta)-\phi(-\theta)$. In fact, if $g_{k}^{(k)}$ corresponds to a probability measure $\left\{p_{i}^{(k)}: i \geq 0\right\}$ then, for each $\theta \in[0,1], g_{k \theta}^{(k)}$ is the generating function of the probability measure

$$
p_{i}^{(k)}(\theta)=p_{i}^{(k)} \mathrm{e}^{-(1-\theta)(i-1) / k}, \quad i \geq 1,
$$

and $p_{0}^{(k)}(\theta)=1-\sum_{i \geq 1} p_{i}^{(k)}(\theta)$.

\section{Acknowledgements}

We would like to give our sincere thanks to Professor Zenghu Li for his encouragement and helpful discussions. The authors also wish to thank an anonymous referee for his/her remarks that significantly improved the presentation of the paper. The work was supported by the NSFC (grant numbers 11126037, 11201030, 11071021) and the Ministry of Education (985 Project).

\section{References}

Abraham, R. and Delmas, J.-F. (2012). A continuum-tree-valued Markov process. Ann. Prob. 40, 1167-1211.

Abraham, R., Delmas, J.-F. and He, H. (2012). Pruning Galton-Watson trees and tree-valued Markov processes. Ann. Inst. H. Poincaré Prob. Statist. 48, 688-705.

Aldous, D. (1978). Stopping times and tightness. Ann. Prob. 6, 335-340.

Aldous, D. (1991). The continuum random tree I. Ann. Prob. 19, 1-28.

Aldous, D. (1993). The continuum random tree III. Ann. Prob. 21, 248-289.

Aldous, D. And Pitman, J. (1998). Tree-valued Markov chains derived from Galton-Watson processes. Ann. Inst. H. Poincaré Prob. Statist. 34, 637-686.

BAKhtin, Y. (2011). SPDE approximation for random trees. Markov Process. Relat. Fields 17, 1-36.

Bertoin, J. AND LE GALL, J.-F. (2006). Stochastic flows associated to coalescent processes III: limit theorems. Illinois J. Math. 50, 147-181.

Dawson, D. A. ANd Li, Z. (2012). Stochastic equations, flows and measure-valued processes. Ann. Prob. 40, 813-857.

Duquesne, T. And Le Gall, J.-F. (2002). Random Trees, Lévy Processes and Spatial Branching Processes (Astérisque 281). SMF, Paris.

Ethier, S. N. And Kurtz, T. G. (1986). Markov Processes: Characterization and Convergence. Wiley, New York.

HE, H. AND MA, R. (2014). Limit theorems for flows of branching processes. Frontiers Math. China 9, 63-79.

Ikeda, N. And Watanabe, S. (1989). Stochastic Differential Equations and Diffusion Processes, 2nd edn. NorthHolland, Amsterdam.

LAMPERTI, J. (1967). The limit of a sequence of branching processes. Z. Wahrscheinlichkeitsth. 7, 271-288.

Le Gall, J.-F. ANd LE JaN, Y. (1998a). Branching processes in Lévy processes: the exploration process. Ann. Prob. 26, 213-252.

LE GALl, J.-F. AND LE JAN, Y. (1998b). Branching processes in Lévy processes: Laplace functionals of snakes and superprocesses. Ann. Prob. 26, 1407-1432.

LI, Z. (2011). Measure-Valued Branching Markov Processes. Springer, Heidelberg.

LI, Z. (2014). Path-valued branching processes and nonlocal branching superprocesses. Ann. Prob. 42, 41-79.

Li, Z. AND MA, C. (2008). Catalytic discrete state branching models and related limit theorems. J. Theoret. Prob. 21, 936-965.

Roelly-Coppoletta, S. (1986). A criterion of convergence of measure-valued processes: application to measure branching processes. Stochastics 17, 43-65. 